\title{
The algebra of functions with Fourier transforms in a given function space
}

\section{U.B. Tewari and A.K. Gupta}

Let $G$ be a locally compact abelian group and $\hat{G}$ be its dual group. For $I \leq p<\infty$, let $A_{p}(G)$ denote the set of all those functions in $L_{1}(G)$ whose Fourier transforms belong to $L_{p}(\hat{G})$. Let $M\left(A_{p}(G)\right)$ denote the set of all functions $\varphi$ belonging to $L_{\infty}(\hat{G})$ such that $\varphi \cdot \hat{f}$ is Fourier transform of an $L^{1}$-function on $G$ whenever $f$ belongs to $A_{p}(G)$. For $1 \leq p<q<\infty$, we prove that $A_{p}(G) \varsubsetneqq A_{q}(G)$ provided $G$ is nondiscrete. As an application of this result we prove that if $G$ is an infinite compact abelian group and $1 \leq p \leq 4$ then $Z_{p}(\hat{G}) \underset{\ddagger}{\subsetneq} M\left(A_{p}(G)\right)$, and if $p>4$ then there exists $\psi \in z_{p}(\hat{G})$ such that $\psi$ does not belong to $M\left(A_{p}(G)\right)$.

\section{Introduction}

Let $G$ be a locally compact abelian group and let $I \leq p<\infty . A_{p}(G)$ is the Banach algebra consisting of all those functions $f \in L_{I}(G)$ for which $\hat{f} \in L_{p}(\Gamma)$ where $\Gamma$ denotes the dual group of $G$. The multiplication in $A_{p}(G)$ is the convolution and the norm is given by

$$
\|f\|^{p}=\|f\|_{I}+\|\hat{f}\|_{p}\left(f \in A_{p}(G)\right) .
$$


In [7] and [8] Larsen stated without proof that the $A_{p}(G)$ are distinct for distinct $p$ unless $G$ is discrete in which case $A_{p}(G)=L_{1}(G), \forall p$. In a private communication Professor Larsen told us that his proof of this assertion was fallacious and he gave a proof of the fact that $A_{p}(G) \varsubsetneqq A_{q}(G), p<q$ in the case that $G$ is $R$ or $T$ or $G$ is infinite compact and $1 \leq p<2$. In this paper we shall prove this result for nondiscrete $G$. As an application we shall show that for an infinite compact abelian group $G, Z_{p}(\Gamma) \underset{\ddagger}{\subsetneq} M\left(A_{p}(G)\right)$, if $I \leq p \leq 4$, and there exists $\psi \in Z_{p}(\Gamma)$ such that $\psi \notin M\left(A_{p}(G)\right)$, if $p>4$. Here $M\left(A_{p}(G)\right)$ denotes the algebra of multipliers of $A_{p}(G)$. For the results on $A_{p}(G)$ and its multipliers we refer the reader to [7], where the standard results from harmonic analysis and functional analysis are also given in the appendices.

\section{Results on $A_{p}(G)$}

In this section we shall prove that for nondiscrete $G$ and $1 \leq p<q<\infty, A_{p}(G) \underset{\varsubsetneqq}{\subsetneq} A_{q}(G)$. The proof of this fact depends on several intermediary results which are of interest for their own sake.

PROPOSITION 1. Let $G$ be a nondiscrete locally compact abelian

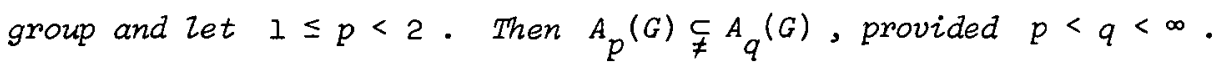

Proof. Since $G$ is nondiscrete, therefore $\Gamma$ is non-compact. Let $U$ be a symmetric neighbourhood of $O$ in $\Gamma$ such that $\bar{U}$ is compact. Choose a sequence $\left\{\gamma_{n}\right\}$ in $\Gamma$ such that $\left(\gamma_{i}+U+U\right)$ is disjoint from $\left(\gamma_{j}+U+U\right)$, unless $i=j$. Let $g=x_{U}$ and

$$
h=\sum_{k=1}^{\infty} \frac{1}{k^{1 / p}} x_{\gamma_{k}+U+U}
$$

(for any set $A, \chi_{A}$ denotes the characteristic function of $A$ ). Then $g$ and $h$ both belong to $L_{2}(\Gamma)$ and hence there exists $f \in L_{1}(G)$ such that $\hat{f}=g \star h$. Moreover, $g \in L_{1}(\Gamma)$ and $h \in L_{q}(\Gamma)$; therefore 
$f \in A_{q}(G)$. But $f \notin A_{p}(G)$. In fact

$$
g * h(\tau)=\frac{1}{k^{1 / p}} \rho(U)
$$

for each $\tau \in \gamma_{k}+U$, where $\rho$ is the Haar measure on $\Gamma$. Since $\left(\gamma_{i}+U\right)$ and $\left(\gamma_{j}+U\right)$ are disjoint for $i \neq j$, it follows that $g * h \leqslant L_{p}(\Gamma)$, that is, $f \notin A_{p}(G)$.

REMARK. The proof of Proposition $I$ is a modification of the arguments of Martin and Yap [9], p. 218.

COROLLARY 1. Let $G=T$, the circle group, and $1 \leq p<q<\infty$. Then $A_{p}(G) \varsubsetneqq A_{q}(G)$.

Proof. If $1 \leq p<2$, then the result follows from Proposition 1 . If $p \geq 2$ then $q>2$ and the conjugate index $q^{\prime}$ lies between $I$ and 2. It is known (Edwards [2], p. 147) that there exists $f \in L_{q^{\prime}}(G)$ such that $\hat{f}$ does not belong to $L_{p}(\Gamma)$. Such a function $f$ belongs to $A_{q}(G)$ but $f \notin A_{p}(G)$.

PROPOSITION 2. Let $G=R$, the real line, and $1 \leq p<q<\infty$. Then $A_{p}(G) \varsubsetneqq A_{q}(G)$.

Proof. Since $p<q$ it follows that $A_{p}(G) \subseteq A_{q}(G)$. Moreover, it can be easily seen that $\|f\|^{q} \leq 2\|f\|^{p}$ for every $f \in A_{p}(G)$. Therefore the assumption that $A_{p}(G)=A_{q}(G)$ would lead to the existence of a constant $K>0$ such that

$$
\|f\|_{1}+\|\hat{f}\|_{p} \leq K\left[\|f\|_{1}+\|\hat{f}\|_{q}\right]
$$

for every $f \in A_{p}(G)$. We shall show that (1) leads to a contradiction. For this purpose consider the function

$$
\Delta_{\alpha}(x)= \begin{cases}1-\frac{|x|}{\alpha}, & |x| \leq \alpha, \\ 0, & |x|>\alpha,\end{cases}
$$

where $\alpha>0$. 
Let $f_{\alpha}=\varangle_{\alpha}$ where denotes the inverse Fourier transform, that is,

$$
f_{\alpha}(x)=\int_{-\infty}^{\infty} \Delta_{\alpha}(t) e^{i t x} d t
$$

Then $\left\|f_{\alpha}\right\|_{1}=2 \pi ;\left(\operatorname{see}[4]\right.$, pp. 21-22). Moreover $\hat{f}_{\alpha}=2 \pi \Delta_{\alpha}$ and

$$
\left\|\hat{f}_{\alpha}\right\|_{p}=2 \pi\left(\frac{2}{p+1}\right)^{1 / p} \alpha^{1 / p} \text { for } 1 \leq p<\infty .
$$

(2) follows from an easy computation. From (1) and (2) it follows that

$$
2 \pi+2 \pi\left(\frac{2}{p+1}\right)^{1 / p} \alpha^{1 / p} \leq K\left[2 \pi+2 \pi\left(\frac{2}{q+1}\right)^{1 / q} \alpha^{1 / q}\right]
$$

or

$$
2 \pi\left(\frac{2}{p+1}\right)^{1 / p} \alpha^{1 / p} \leq(K-1) 2 \pi+2 K \pi\left(\frac{2}{q+1}\right)^{1 / q} \alpha^{1 / q} \text {. }
$$

Dividing (3) by $\alpha^{1 / q}$ on both sides we get

$$
2 \pi\left(\frac{2}{p+1}\right)^{1 / p} \alpha^{1 / p-1 / q} \leq(K-1) 2 \pi \alpha^{-1 / q}+2 K \pi\left(\frac{2}{q+1}\right)^{1 / q} .
$$

Taking limit as $\alpha+\infty$ in (4) we see that the right hand side of (4) remains bounded while the left hand side tends to $\infty$ since $p<q$. This contradiction establishes the proposition.

PROPOSITION 3. Let $G$ be an infinite compact totally disconnected abelian group and $1 \leq p<q<\infty$. Then $A_{p}(G) \varsubsetneqq A_{q}(G)$.

Proof. As in the proof of Proposition 2, the assumption that $A_{p}(G)=A_{q}(G)$ would lead to the existence of a constant $K>0$ such that

$$
\|f\|_{1}+\|\hat{f}\|_{p} \leq K\left[\|f\|_{1}+\|\hat{f}\|_{q}\right]
$$

for every $f \in A_{p}(G)$. Then we shall have

$$
\|\hat{f}\|_{p} \leq K\|f\|_{1}+K\|\hat{f}\|_{q} \text { for every } f \in A_{p}(G) \text {. }
$$

We shall show that (5) leads to a contradiction. Since $G$ is compact and totally disconnected, there exists a neighbourhood basis $\left\{V_{\alpha}\right\}_{\alpha \in I}$ of 
0 in $G$ consisting of open and closed subgroups of $G$; (see [5],

p. 62). Since $G$ is infinite compact, it follows that

$$
\lim _{\alpha} \lambda\left(v_{\alpha}\right)=0 \text {, }
$$

where $\lambda$ denotes the normalized Haar measure on $G$.

Let $\lambda_{\alpha}=\lambda\left(V_{\alpha}\right)$ and let $X_{\alpha}$ denote the annihilator subgroup of $V_{\alpha}$. Since $V_{\alpha}$ is open and closed, it follows that $X_{\alpha}$ is finite. Let $n_{\alpha}$ be the number of points in $x_{\alpha}$ and let $f_{\alpha}=\chi_{V_{\alpha}}$. Then $\left\|f_{\alpha}\right\|_{1}=\lambda_{\alpha}$ and $\hat{f}_{\alpha}=\lambda_{\alpha} X_{X_{\alpha}}$. Also $\left\|f_{\alpha}\right\|_{2}^{2}=\lambda_{\alpha}$. Therefore by the Plancherel Theorem we get

$$
\lambda_{\alpha}=\left\|f_{\alpha}\right\|_{2}^{2}=\sum_{\gamma \in \Gamma}|\hat{f}(\gamma)|^{2}=\lambda_{\alpha}^{2} \cdot n_{\alpha}
$$

Therefore $n_{\alpha}=1 / \lambda_{\alpha}$. Now, for $1 \leq p<\infty$, we have $\left\|\hat{f}_{\alpha}\right\|_{p}=\lambda_{\alpha} \cdot n_{\alpha}^{1 / p}=\lambda_{\alpha}^{1 / p^{\prime}}$, where $1 / p+1 / p^{\prime}=1$.

From (5) it follows that

$$
\lambda_{\alpha}^{1 / p^{\prime}} \leq K \lambda_{\alpha}+\lambda_{\alpha}^{1 / q^{\prime}}
$$

Divioing both sides of (7) by $\lambda_{\alpha}^{l / q^{\prime}}$ we get

$$
\lambda_{\alpha}^{1 / p^{\prime}-1 / q^{\prime}} \leq K \lambda_{\alpha}^{1 / q}+K
$$

Taking the limit in $(8)$ we see that the right hand side remains bounded, while the left hand side tends to $\infty$, because $1 / p^{\prime}<1 / q^{\prime}$ and $\lambda_{\alpha}$ tends to zero. This contradiction yields the proof.

REMARK. The above proof uses a technique due to Edwards [3], p. 196.

PROPOSITION 4. Let $G_{1}$ and $G_{2}$ be locally compact abelian groups and $G=G_{1} \times G_{2}$. Let $1 \leq p<q<\infty$. If $A_{p}\left(G_{i}\right) \neq A_{q}\left(G_{i}\right)$ for some $i$, then $A_{p}(G) \neq A_{q}(G)$. 
Proof. We may assume that $A_{p}\left(G_{1}\right) \neq A_{q}\left(G_{1}\right)$ (the proof in the other case is exactly similar $)$. Choose $f \in A_{q}\left(G_{1}\right)$ such that $f \notin A_{p}\left(G_{1}\right)$. Let $g$ be any non-zero function belonging to $A_{p}\left(G_{2}\right)$. Define

$$
h(x, y)=f(x) \cdot g(y) \text { for }(x, y) \in G_{1} \times G_{2} .
$$

Then $h \in A_{q}(G)$ but $h \notin A_{p}(G)$, because $\hat{h}(r, n)=\hat{f}(r) \cdot \hat{g}(n)$ and $\hat{h} \in L_{p}(\Gamma)$ if and only if $\hat{f} \in L_{p}\left(\Gamma_{1}\right)$ and $\hat{g} \in L_{p}\left(\Gamma_{2}\right)$, where $\Gamma, \Gamma_{1}$ and $\Gamma_{2}$ are the dual groups of $G, G_{1}$ and $G_{2}$ respectively.

Let $G$ be a locally compact abelian group and let $H$ be a closed subgroup. For a continuous function $f$ on $G$ which has compact support, define

$$
\pi_{H}(f)(x)=\int_{H} f(x y) d y
$$

where $d y$ denotes Haar integral on $H$. It is well known (see [10], Chapter 3) that $\pi_{H}(f)$ is constant on cosets modulo $H$ and that $\pi_{H}(f)$ defines $\&$ continuous function on $G / H$ which has compact support. This gives a mapping $\pi_{H}$ from $C_{C}(G)$ into $C_{C}(G / H)$, where $C_{C}(G)$ denotes the space of continuous functions with compact support. This mapping $\pi_{H}$ extends to $L_{1}(G)$ and it maps $L_{1}(G)$ onto $L_{1}(G / H)$. Reiter [11] has shown that if $S(G)$ is a Segal algebra on $G$, then $\pi_{H}(S(G)$ ) becomes a Segal algebra on $G / H$. The following proposition is interesting because it shows that $\pi_{H}\left(A_{p}(G)\right)=A_{p}(G / H)$ under the hypothesis that $H$ is compact. We shall use this proposition to prove Theorem $I$ of this paper.

PROPOSITION 5. Let $G$ be a locally compact abelian group and let $H$ be a compact subgroup of $G$. Then $\pi_{H}\left(A_{p}(G)\right)=A_{p}(G / H)$.

Proof. Let $\Lambda$ be the annihilator of $H$. Since $H$ is compact, it follows that $\Lambda$ is open. Also for $f \in L_{1}(G), \pi_{H}(f)^{\wedge}=\left.\hat{f}\right|_{\Lambda}$. Therefore $f \in A_{p}(G)$ implies that $\pi_{H}(f) \in A_{p}(G / H)$ and hence $\pi_{H}\left(A_{p}(G)\right) \subseteq A_{p}(G / H)$. To prove the other inclusion take $f^{\prime} \in A_{p}(G / H)$. Then there exists 
$f \in L_{\perp}(G)$ such that $\pi_{H}(f)=f^{\prime}$. Consider the function $g=m_{H} * f$ where $m_{H}$ is the normalized Haar measure on $H$ and $m_{H}$ is considered as a bounded measure on the whole group $G$ in the usual manner. Since $\hat{m}_{H}=x_{\Lambda}$ it follows that $\pi_{H}(g)=f^{\prime}$, because $\pi_{H}(g)$ and $f^{\prime}$ have the same Fourier transform. Also since $\Lambda$ is open and $\hat{g}=\hat{f}^{\prime}$ on $\Lambda$ and $\hat{g}=0$ outside $\Lambda$, it follows that $g \in A_{p}(G)$. Therefore $\pi_{H}\left(A_{p}(G)\right)=A_{p}(G / H)$.

THEOREM 1. Let $G$ be an infinite compact abelian group and let $1 \leq p<q<\infty$. Then $A_{p}(G) \underset{q}{\subsetneq} A_{q} G$.

Proof. We have already proved the theorem for totally disconnected $G$ in Proposition 3. Let us now suppose that $G$ is not totally disconnected. Then the dual group $\Gamma$ has an element of infinite order; (see [12], p. 47). Therefore $\Gamma$ contains $Z$ (the group of integers) as a subgroup. Let $H$ be the annihilator of this subgroup. Then the dual of $G / H$ is isomorphic to $Z$ and hence $G / H$ is isomorphic to $T$ (the circle group). By Corollary 1 it follows that $A_{p}(G / H) \underset{\ddagger}{\subsetneq} A_{q}(G / H)$. The theorem now follows from Proposition 5.

COROLLARY 2. Let $G$ be a nondiscrete locally compact, compactly generated abelian group and $1 \leq p<q<\infty$. Then $A_{p}(G) \underset{\ddagger}{\subset} A_{q}(G)$.

Proof. From Theorem 9.8 of [5] it follows that $G=R^{a} \times z^{b} \times F$, where $a$ and $b$ are nonnegative integers and $F$ is a compact abelian group. If $a>0$ then the result follows from Propositions 2 and 4 . If $a=0$ then since $G$ is nondiscrete it follows that $F$ is an infinite compact abelian group. The result then follows from Theorem 1 and Proposition 4.

PROPOSITION 6. Let $G$ be a locally compact abelian group and let $H$ be an open subgroup of $G$. Let $f \in L_{1}(H)$. Define $g$ on $G$ by setting $g=f$ on $H$ and $g=0$ outside $H$. Then $g \in L_{1}(G)$ and $\hat{g} \in L_{p}(\Gamma)$ if and only if $\hat{f} \in L_{p}(\Gamma / \Lambda)$, where $1 \leq p<\infty$ and $\Lambda$ is the annihilator of $H$. 
Proof. It is obvious that $g \in L_{1}(G)$. Let $F$ be the set of all those elements $\varphi \in L_{1}(\Gamma)$ which are almost everywhere constant on each coset of $\Lambda$. Let $\eta: \Gamma \rightarrow \Gamma / \Lambda$ be the quotient map. Then it follows from Theorem 28.55 of [6] that $h \rightarrow h \circ \eta$ is a Banach algebra isomorphism of $L_{1}(\Gamma / \Lambda)$ onto $F^{\cdot}$. Since $g$ is zero outside $H, \hat{g}$ is constant on cosets of $\Lambda$. Moreover $\hat{g}=\hat{f} \circ \eta$ and $|\hat{g}|^{p}=|\hat{f}|^{p} \circ \eta$. Hence it follows that $\hat{g} \in L_{p}(\Gamma)$ if and only if $\hat{f} \in L_{p}(\Gamma / \Lambda)$.

COROLLARY 3. Let $G$ be a locally compact abelian group and $1 \leq p<q<\infty$. Let $H$ be an open subgroup of $G$ such that $A_{p}(H) \underset{\ddagger}{\subset} A_{q}(H)$. Then $A_{p}(G) \underset{\ddagger}{A_{q}}(G)$.

Proof. Let $f \in A_{q}(H)$ such that $f \vDash A_{p}(H)$. Define $g$ as in Proposition 6. Then Proposition 6 implies that $g \in A_{q}(G)$ but $g \notin A_{p}(G)$.

THEOREM 2. Let $G$ be a nondiscrete Zocally-compact abelian group and $i \leq p<q<\infty$. Then $A_{p}(G) \underset{\ddagger}{ } A_{q}(G)$.

Proof. Theorem 2.4.1 of [12] implies that there exists an open subgroup $H$ of $G$ such that $H=R^{n} \times F$ where $n$ is a nonnegative integer and $F$ is a compact abelian group. If $n>0$ then $A_{p}(H) \underset{\neq}{\subsetneq} A_{q}(H)$ by Proposition 4. If $n=0$ then since $G$ is nondiscrete and $H$ is open it follows that $F$ is an infinite compact abelian group. Therefore

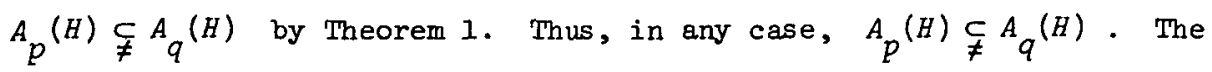
proof now follows from Corollary 3.

\section{Multipliers of ${ }^{A} p(G)$}

In this section $G$ will denote an infinite compact abelian group and $\Gamma$ its dual group. We shall prove:

THEOREM 3. $Z_{p}(\Gamma) \varsubsetneqq M\left(A_{p}(G)\right)$ for $1 \leq p \leq 4$. If $4<p<\infty$ then there exists $\psi \in Z_{p}(\Gamma)$ such that $\psi \notin M\left(A_{p}(G)\right)$.

Proof. It is known that every bounded function on $\Gamma$ defines a 
multiplier on $A_{p}(G)$, provided $1 \leq p \leq 2$ (see [7], p. 207). Therefore we may assume that $p>2$. Suppose $p \leq 4$ and $\varphi \in l_{p}(\Gamma)$ and $f \in A_{p}(G)$. By Hölder's inequality we have

$$
\begin{aligned}
\sum_{\gamma}|\varphi \hat{f}(\gamma)|^{2} & \leq\left(\sum_{\gamma}|\varphi(\gamma)|^{2 p / p-2}\right)^{I-2 / p}\left(\sum_{\gamma}|\hat{f}(\gamma)|^{p}\right\}^{2 / p} \\
& <\infty,
\end{aligned}
$$

because $\varphi \in l_{p}(\Gamma)$ and $\frac{2 p}{p-2} \geq p$ for $2<p \leq 4$. Therefore there exists $g \in L_{2}(G)$ such that $\hat{g}=\varphi \cdot \hat{f}$. Clearly $g \in A_{p}(G)$. Therefore

$\varphi \in M\left(A_{p}(G)\right)$. Since the constant sunctions on $\Gamma$ define multipliers on $A_{p}(G)$ it follows that $I_{p}(\Gamma) \underset{\ddagger}{\subset} M\left(A_{p}(G)\right]$.

Let us now consider the case when $4<p<\infty$. By Theorem 1, there exists a function $f \in L_{1}(G)$ such that $\sum_{\gamma}|\hat{f}(\gamma)|^{4}=\infty$ and $\sum_{\gamma \in \Gamma}|\hat{f}(\gamma)|^{p}<\infty$. Let $\varphi=\hat{f}$. Then $\varphi \leqslant z_{p}(\Gamma)$. However $\varphi^{2} \mid z_{2}(\Gamma)$. Therefore, from Theorem 1.1 of [1] it follows that there exists a function $\varepsilon$ on $\Gamma$ such that $\varepsilon(\gamma)= \pm I$ and for no integrable function $g$ on $G$ we have $\hat{g}(\gamma)=\varepsilon(\gamma) \varphi(\gamma) \cdot \varphi(\gamma)$. Now the function $\psi(\gamma)=\varepsilon(\gamma) \varphi(\gamma)$ is a function belonging to $z_{p}(\Gamma)$, but $\psi \cdot \hat{f}$ is not Fourier transform of any integrable function. Therefore $\psi \notin M\left(A_{p}(G)\right)$ even though $\psi \in z_{p}(\Gamma)$.

\section{References}

[1] R.E. Edwards, "Changing signs of Fourier coefficients", Pacific $J$. Math. 15 (1965), 463-475.

[2] R.E. Edwards, Fourier semies; A modem introduction, Volume II (Holt, Rinehart and Winston, New York, Chicago, San Francisco, Atlanta, Dellis, Montreal, Toronto, London, 1967).

[3] R.E. Edwards, "Inequalities related to those of Hausdorff-Young", Bul2. Austral. Math. Soc. 6 (1972), 185-210. 
[4] P.R. Goldberg, Fourier transforms (Cambridge Tracts in Mathematics and Mathematical Physics, 52. Cambridge University Press, Cambridge, 1961).

[5] Edwin Hewitt and Kenneth A. Ross, Abstract harmonic analysis, Volume I (Die Grundlehren der mathematischen Wissenschaften, Band 115. Academic Press, New York; Springer-Verlag, Berlin, Göttingen, Heidelberg; 1963).

[6] Edwin Hewitt and Kenneth A. Ross, Abstract harmonic analysis, Volume II (Die Grundlehren der mathematischen Wissenschaften, Band 152. Springer-Verlag, Berlin, Heidelberg, New York, 1970).

[7] Ronald Larsen, An introduction to the theory of multipliers (Die Grundlehren der mathematischen Wissenschaften, Band 175. Springer-Verlag, Berlin, Heidelberg, New York, 1971).

[8] Ronald Larsen, "The multipliers for functions with Fourier transforms in $L_{p}$ ", Math. Scand. 28 (1971), 215-225.

[9] John C. Martin and Leonard Y.H. Yap, "The algebra of functions with Fourier transforms in $L^{p} "$, Proc. Amer. Math. Soc. 24 (1970), 217-219.

[10] Hans Reiter, Classical harmonic analysis and locally compact groups (Clarendon Press, Oxford, 1968).

[11] Hans Reiter, $L^{1}$-algebras and Segal algebras (Lecture Notes in Mathematics, 231. Springer-Verlag, Berlin, Heidelberg, New York, 1971).

[12] Walter Rudin, Fourier analysis on groups (Interscience, New York, London, 1962).

Department of Mathematics, Indian Institute of Technology, Kanpur, India. 